\title{
The role of $\alpha$-satellite DNA and heterochromatin polymorphism in leukemia patients and illicit drug addicts
}

\author{
A. Movafagh ${ }^{1}$, S.A. Mortazavi-Tabatabaei ${ }^{2}$ and A.A. Kolahi ${ }^{3}$ \\ ${ }^{1}$ Department of Medical Genetics, Shahid Beheshti University of Medical Sciences, \\ Tehran, Iran \\ ${ }^{2}$ Proteomics Research Center, Shahid Beheshti University of Medical Sciences, \\ Tehran, Iran \\ ${ }^{3}$ Department of Health and Community Medicine, \\ Shahid Beheshti University of Medical Sciences, Tehran, Iran
}

Corresponding author: A. Movafagh

E-mail: Movafagh_a@yahoo.com

Genet. Mol. Res. 10 (4): 3999-4005 (2011)

Received August 19, 2011

Accepted October 21, 2011

Published November 25, 2011

DOI http://dx.doi.org/10.4238/2001.November.25.3

\begin{abstract}
Heterochromatin is considered to play a role in protecting the genome against mutagens. Changes in the quantity and proportion of different types of satellite DNA could increase genetic susceptibility in individuals with heterochromatic variations; they cause chromosome instability and predispose patients to malignancies. We evaluated the heterochromatin associated with chromosomes in 50 leukemia patients, 93 drug addicts and 93 healthy controls from Tehran, Iran. Barium hydroxide saline Giemsa staining was used to examine heterochromatin polymor-
\end{abstract}


phism of chromosomes 1, 9 and 16 in lymphocyte cultures. There were significant differences in this polymorphism in lymphocytes from drug addicts and leukemia patients compared to healthy controls. These polymorphisms could serve as markers for the detection and characterization of chromosome damage in leukemia patients and drug addicts.

Key words: Addiction; Drugs; Leukemia; Heterochromatin; Polymorphism

\section{INTRODUCTION}

Eukaryotic genomes contain far more DNA than needed for coding proteins. Some of these additional DNA sequences comprise non-coding repetitive DNA sequences, mostly satellite DNAs and also transposable elements, usually located in the heterochromatic regions of chromosomes. Satellite DNAs consist of tandem repeated DNA sequences inhabiting the mammalian genome, typically organized in long arrays of hundreds or thousands of copies. Different important functions have been ascribed to satellite DNA, from the imperative centromeric function in mitosis and meiosis to the recent discovery of its involvement in regulatory functions via satellite transcripts. Moreover, satellite DNAs, among other repetitive sequences, are believed to be the "engine" triggering the mammalian genome (Sivakumaran et al., 1997; Grewal and Moazed, 2003). Heterochromatin mediates many diverse functions in the cell nucleus, including centromere function, gene silencing, regulation of gene expression, and nuclear organization (Cortés et al., 2003). This study represents the first cytogenetic study of heterochromatin polymorphism regions of chromosomes 1, 9 and 16 together, ever conducted in Iranians and reported for a Middle East population. Hence, the aim of the present study was to evaluate the role of heterochromatin polymorphism associated with chromosomes in addicts and leukemia patients compared to healthy normal controls.

\section{MATERIAL AND METHODS}

A study of variant heterochromatin of chromosomes 1, 9 and 16 was carried out by C-banding in peripheral blood lymphocyte cultures from a total of 50 leukemia patients, including all sub-classifications of AML according to the French American British (FAB) classification. The patients included 16 males and 19 females, with an age range of 18 to 64 years, $(38.11 \pm 15.75$; mean $\pm \mathrm{SD})$, seen at the Modares and Taleghani major referral hospitals in Tehran, Iran. The controls consisted of 50 randomly selected healthy individuals, with an age range of 18 to $49(28.59 \pm 6.87)$. Also, a study of the variant heterochromatin of chromosomes 1, 9, and 16 was performed by C-banding in lymphocyte cultures from a total of 93 drug addicts (addicted to heroin, opium, hasheesh, etc.) and 93 healthy controls. Peripheral blood sample of addicts were collected from the Loghman referral hospital and some private hospitals in Tehran, Iran. The age of the drug addicts ranged from 17 to 75 years and the mean was $32.4 \pm 11.4$. The control group consisted of 93 randomly selected healthy adult persons (their ages ranged from 18 to 48 , with a mean of $29.2 \pm 6.8$ ). 


\section{Cytogenetics}

Approximately $0.5 \mathrm{~mL}$ of peripheral blood was obtained from each participant. The blood sampling and cell culture procedures were essentially the same for all the participants. Briefly, heparanized blood was immediately mixed with 4 mL RPMI-1640 (Gibco BRL, USA) cell culture medium supplemented with $15-20 \%$ heat-inactivated fetal bovine serum (Gibco BRL), $100 \mathrm{mg} / \mathrm{mL}$ phytohemagglutinin (PHA) in a Vacutainer tube (Becton Dickinson Co, Ltd USA). This tube was cultured for $70 \mathrm{~h}$ at $37^{\circ} \mathrm{C}$ and incubated with $5 \% \mathrm{CO}_{2}$. After the an incubation period, the cultured cell were harvested by $0.1 \mathrm{~mL}$ with $75 \mathrm{~mL} 10 \mu \mathrm{g} / \mathrm{mL}$ colcemid (Gibco BRL) and incubated at $37^{\circ} \mathrm{C}$ for $30 \mathrm{~min}$. The content of the tube was then centrifuged for $10 \mathrm{~min}$ at $1000 \mathrm{rpm}$ and resuspended in $10 \mathrm{~mL} 75 \mathrm{mM} \mathrm{KCl}$ (Sigma, Co) prewarmed to $37^{\circ} \mathrm{C}$ for $20 \mathrm{~min}$. At this stage, $1 \mathrm{~mL} 20^{\circ} \mathrm{C}$ Carnoys fixative $(3: 1$ methanol:acetic acid; Fisher Scientific) was added to the tube to stop further cell swelling. This fixation was repeated four times. The cells were then dropped onto clean slides, and incubated for 3 days at $60^{\circ} \mathrm{C}$ on a slide warmer. The barium hydroxide/saline/Giemsa (BSG) method, with some alterations, was applied (Sumner, 1982). Chromosome preparations were treated with $0.2 \mathrm{~N} \mathrm{HCl}$ for $1 \mathrm{~h}$ at room temperature, followed by a rinse with deionized water. The slides were placed in a freshly prepared $5 \%$ aqueous solution of barium hydroxide octahydrate $\left[\left(\mathrm{Ba}(\mathrm{OH})_{2} .8 \mathrm{H}_{2} \mathrm{O}\right]\right.$ at $56^{\circ} \mathrm{C}$ in a water bath (WB) for about $2-5 \mathrm{~min}$, followed by rinsing with deionized water. Slides were incubated for $1 \mathrm{~h}$ at $60^{\circ} \mathrm{C}$ (WB) in $2 \mathrm{X} \operatorname{SSC}(0.3 \mathrm{M}$ sodium chloride containing $0.03 \mathrm{M}$ tri-sodium citrate) followed by rinsing with water. The treated chromosomes were stained with Giemsa for about $45 \mathrm{~min}$. A minimum of eighty well-spread metaphases were photographed from each individual. To eliminate the variations in C-segment lengths in chromosomes 1, 9 and 16 , the presence of heterochromatin variants were estimated visually when at least $25 \%$ variation in C-band size was observed between homologous chromosomes.

Heterochromatin region differences of the abnormal C-block were recorded as $\mathrm{qh}^{+}$or $\mathrm{qh}$ - Two groups of localization of $\mathrm{C}$-segment inversion were distinguished as total inversion when the whole $\mathrm{C}$-segment inversion was distinguished as total inversion when the whole $\mathrm{C}$ segment was situated near the centromere, but on the short (p) and partly on the long (q) arm of the chromosome. In routine practice, $15 \mathrm{G}$-banded metaphases from each preparation were needed for scoring. In some cases this was not possible, while in others more metaphases were analyzed for better definition of a particular aberration. Well-spread metaphases were saved by the Yvisis software and karyotypes were described according to ISCN (Brothman et al., 2009).

\section{Statistical analysis}

The results of the investigation were statistically analyzed by applying the chi-square test and the Fisher exact test, where the statistical differences yielding $\mathrm{P}<0.05$ were considered significant. Data analysis was performed by the SPSS software (version 16, USA).

\section{Ethics statement}

This study was approved by the Institutional Review Board Ethics Committee of Research Deputy, Shahid Beheshti, University of Medical Sciences, Tehran, Iran (Approval No. 3/712-3/2607-7012). After the protocol was approved, written informed consent was obtained 
from all patients/addicts before they entered the study. All data collected from this study were kept confidential. The authors declare that they have no conflict of interests.

\section{RESULTS}

This study dealt with the proportion and analysis of constitutive heterochromatin polymorphism in chromosomes 1,9 and 16 and the frequency of inversions in leukemia patients (Table 1), as well as the healthy controls (Figure 1).

Table 1. Distribution of heterochromatin polymorphism in chromosomes 1,9 and 16 in leukemia and control groups.

\begin{tabular}{lcccccc}
\hline Group & $1 \mathrm{qh}^{+} / \mathrm{q}^{-}$ & $9 \mathrm{q}^{+} / \mathrm{q}^{-}$ & $16 \mathrm{q}^{+} / \mathrm{q}^{-}$ & inv1qh & inv9qh & inv16qh \\
\hline Leukemia & $23(46.0)$ & $19(38.0)$ & $8(16.0)$ & $2(4.0)$ & $1(2.0)$ & $2(4.0)$ \\
Control & $4(8.0)$ & $5(10.0)$ & $1(2.0)$ & $1(2.0)$ & $0(0)$ & $0(0)$ \\
\hline
\end{tabular}

Frequency of variants (\%)

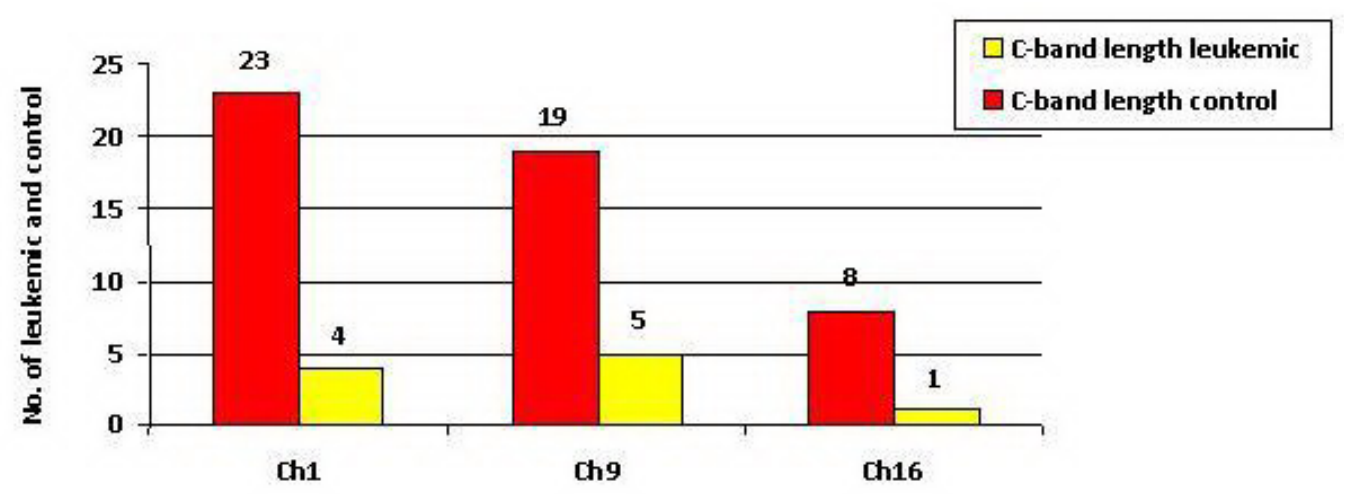

Figure 1. Distribution of C-band length differences in chromosomes no. 1, 9, and 16 between leukemic patients and control.

Constitutive heterochromatin variants of chromosome 1 were detected in $46.0 \%$ of leukemia patients and $8.0 \%$ of the healthy control group. The results revealed significant differences of variant heterochromatin between leukemia patients and the control group $(\mathrm{P}<$ 0.001 ). The differences were significant for chromosome 9 , where variants were $38.0 \%$ in the leukemia patients and $10.0 \%$ in the control group $(\mathrm{P}<0.002)$. The differences were also significant for chromosome 16 , where they were $16.0 \%$ in the leukemia patients and $2.0 \%$ in the control group $(\mathrm{P}<0.04)$. The frequency of partial and complete inversions did not show any significant differences between the leukemia patients and the control group (Table 1).

The proportion of individuals with quantitative heterochromatin size of the homologous chromosome in each of chromosomes 1,9 and 16 and also the relative heterochromatin in drug addicts was compared to the control group shown in Figure 2.

Constitutive heterochromatin variants of chromosome 1 were detected in $17.2 \%$ of addicts and $4.3 \%$ of the control healthy group, and the results revealed statistically significant differences for variant heterochromatin between addicts and the control group $(\mathrm{P}<0.004)$. The differences were significant for chromosome 9 , where they were $9.6 \%$ in addicts and $2.1 \%$ in 
the control group $(\mathrm{P}<0.03)$. The differences were also significant for chromosome 16, where they were $12.9 \%$ in addicts and $4.3 \%$ in the control group $(\mathrm{P}<0.04)$. The frequency of partial and complete inversion did not show any significant differences between the addicts and the control groups (Table 2).

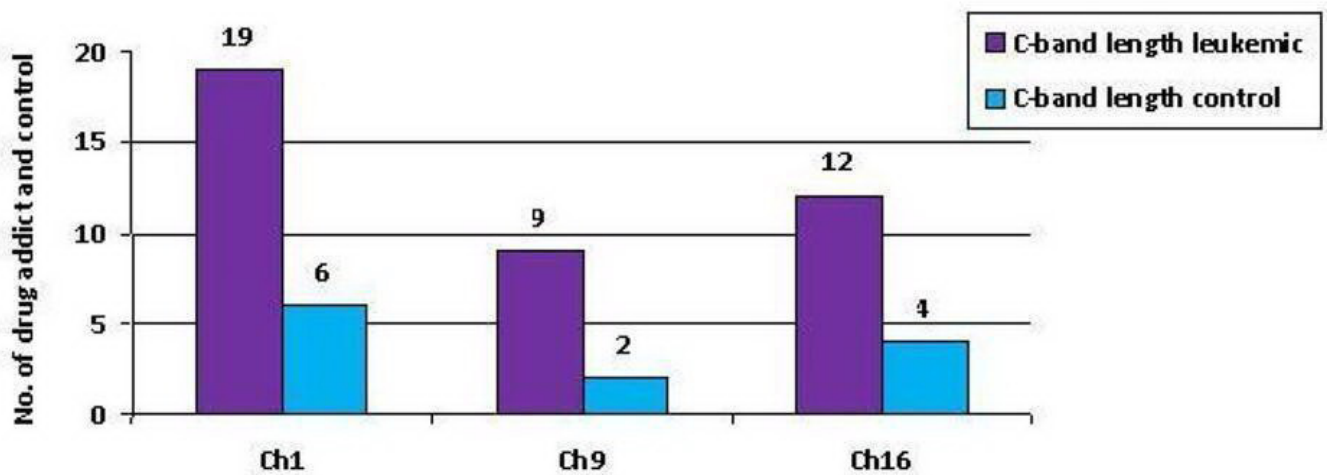

Figure 2. Distribution of C-band length differences in chromosomes no. 1, 9, and 16 between drug addicts and control.

Table 2. Distribution of heterochromatin polymorphism in chromosomes 1, 9 and 16, drug addicts and controls.

\begin{tabular}{lcccccc}
\hline Group & 1qh+/qh- & 9qh+/qh- & $16 \mathrm{q}+/ \mathrm{q}-$ & inv1qh & inv9qh & inv16qh \\
\hline Addicts & $16(17.2)$ & $9(9.6)$ & $12(12.9)$ & $3(3.9)$ & $4(4.3)$ & $3(3.2)$ \\
Control & $4(4.3)$ & $2(2.1)$ & $4(4.3)$ & $1(1.1)$ & $0(0)$ & $1(1.1)$ \\
\hline
\end{tabular}

Frequency of variants (\%)

\section{DISCUSSION}

Heterochromatin is considered to play a role in protecting the genome against mutagens. Changes in the quantity and proportion of the different types of satellite DNA may increase genetic susceptibility in people with heterochromatin variations, which in turn cause chromosome instability and predispose the individual to cancers and malignancies (Sivakumaran et al., 1997). Various surveys have shown an increased frequency of heterochromatin polymorphisms in patients with different disorders, suggesting their possible role in the development of neoplasia (Atkin and Brito-Babapulle, 1981; Ranni et al., 1987).

In the course of this study, an excess of heterochromatin polymorphism of chromosomes 1, 9 and 16 was found in drug addicts compared to the controls. This result is in agreement with previous investigators, who suggested the role of heterochromatin variants of chromosome 1 and the possible risk of developing malignancies (Le Coniant et al., 1982; Berger et al., 1985). Furthermore, the results of this study exhibited an increase in C-band heterochromatin variants in chromosome 9 and 16 in addicts when compared to controls. Therefore, exposure to these drugs, narcotics and perhaps other environmental agents may cause heterochromatin variants and/or chromosomal damages (Bennett et al., 2010). More data are needed in this field (narcotics) to draw a firm conclusion. 
A number of reports have indicated pronounced heteromorphism in size and localization in the C-band region of chromosome 1,9 and 16 in individuals with different malignancies, such as polycythemia vera (Atkin and Brito-Babapulle, 1981; Ranni et al., 1987), male infertility (Madon et al., 2005), ataxia telangiectasia (Grewal and Moazed, 2003), various types of leukemias (Rivera et al., 1999; Enukashvily et al., 2007), and cancers such as breast cancer (Berger et al., 1985; Tsezou et al., 1993; Tsuda et al., 2002; Plohl et al., 2008), retinoblastoma (Sivakumaran et al., 1997), and colon carcinoma (Neglia et al., 2003).

In the course of this study, an excess of constitutive C-band heterochromatin polymorphisms of chromosome 1, 9 and 16 was found in leukemia patients when compared to the control group. This is in agreement with previous studies suggesting the role of heterochromatin variants of chromosome 1, 9 and 16 and possible risk of developing cancer and leukemia (Rivera et al., 1999; Pluth et al., 2000; Pujol et al., 2006; de Assis et al., 2009; National Toxicology Program, 2010; Rossnerova et al., 2010). On the other hand, other investigators could not find any correlation between C-band heterochromatin polymorphism of chromosome 1,9 and 16 and cancer susceptibility (Rey et al., 1987). The present investigation and other studies together may in any case be helpful in understanding the possible predisposing the role of heterochromatin variation in regard to cancer, leukemia and narcotic risk. This study showed that heterochromatin polymorphism of chromosomes 1,9 and 16 may serve as a marker for the detection and characterization of damage to the genome and chromosomes in leukemia and drug addicts.

\section{ACKNOWLEDGMENTS}

We thank Dr. Fatemeh Isfahani, Dr. Abbas Hajfathali, Dr. Hamid Attarian, Dr. Morteza Ghadiani, and Dr. Hamid Rezvani of the Departments of Medical Oncology and Internal Medicine. We are grateful to Dr. Housein Hassani-Moghadam, Department of Community Medicine, Loghma Hospital, for his support and many helpful discussions. We thank Dr. Fariboorz Rashid, Alberta Medical University, Canada, Dr. Hemad Yassaei Auxbridge Medical University, London, for their collaboration and editorial assistance. Also, Miss Niloofar Safavi provided excellent technical assistance and helped with material collection.

\section{REFERENCES}

Atkin NB and Brito-Babapulle V (1981). Heterochromatin polymorphism and human cancer. Cancer Genet. Cytogenet. 3: $261-272$.

Bennett LM, Wang Y, Ramsey MJ, Harger GF, et al. (2010). Cigarette smoking during pregnancy: chromosome translocations and phenotypic susceptibility in mothers and newborns. Mutat. Res. 696: 81-88.

Berger R, Bernheim A, Kristoffersson U, Mitelman F, et al. (1985). C-band heteromorphism in breast cancer patients. Cancer Genet. Cytogenet. 18: 37-42.

Brothman AR, Persons DL and Shaffer LG (2009). Nomenclature evolution: Changes in the ISCN from the 2005 to the 2009 edition. Cytogenet. Genome Res. 127: 1-4.

Cortés A, Huertas D, Marsellach FX, Ferrer-Miralles N, et al. (2003). Analysing the contribution of nucleic acids to the structure and properties of centric heterochromatin. Genetica 117: 117-125.

de Assis KR, Ladeira MS, Bueno RC, Dos Santos BF, et al. (2009). Genotoxicity of cigarette smoking in maternal and newborn lymphocytes. Mutat. Res. 679: 72-78.

Enukashvily NI, Donev R, Waisertreiger IS and Podgornaya OI (2007). Human chromosome 1 satellite 3 DNA is decondensed, demethylated and transcribed in senescent cells and in A431 epithelial carcinoma cells. Cytogenet. Genome Res. 118: 42-54.

Grewal SI and Moazed D (2003). Heterochromatin and epigenetic control of gene expression. Science 301: 798-802. 
Le Coniant M, Vecchione D, Bernheim A and Berger R (1982). C-banding studies in acute nonlymphocytic leukemia. Cancer Genet. Cytogenet. 5: 327-331.

Madon PF, Athalye AS and Parikh FR (2005). Polymorphic variants on chromosomes probably play a significant role in infertility. Reprod. Biomed. Online 11: 726-732.

National Toxicology Program (2010). Final report on carcinogens background document for formaldehyde. Rep. Carcinog. Backgr. Doc. (10-5981): i-512.

Neglia M, Bertoni L, Zoli W and Giulotto E (2003). Amplification of the pericentromeric region of chromosome 1 in a newly established colon carcinoma cell line. Cancer Genet. Cytogenet. 142: 99-106.

Plohl M, Luchetti A, Mestrovic N and Mantovani B (2008). Satellite DNAs between selfishness and functionality: structure, genomics and evolution of tandem repeats in centromeric (hetero)chromatin. Gene 409: 72-82.

Pluth JM, Ramsey MJ and Tucker JD (2000). Role of maternal exposures and newborn genotypes on newborn chromosome aberration frequencies. Mutat. Res. 465: 101-111.

Pujol A, Benet J, Staessen C, Van AE, et al. (2006). The importance of aneuploidy screening in reciprocal translocation carriers. Reproduction 131: 1025-1035.

Ranni NS, Labal VM, Mudry de PM, Slavutsky I, et al. (1987). Heterochromatic variants and their association with neoplasias: III. Multiple myeloma. Cancer Genet. Cytogenet. 28: 101-105.

Rey JA, Bello MJ, de Campos JM, Kusak ME, et al. (1987). C-band pattern in patients with nervous system tumors. Cancer Genet. Cytogenet. 27: 185-190.

Rivera H, Gutierrez-Angulo M and Gonzalez-Garcia JR (1999). Chromosome 9qh inversions may not be true inversions. Hum. Genet. 105: 181-184.

Rossnerova A, Balascak I, Rossner P, Jr. and Sram RJ (2010). Frequency of chromosomal aberrations in Prague mothers and their newborns. Mutat. Res. 699: 29-34.

Sivakumaran TA, Ghose S, Kumar H, Singha U, et al. (1997). Absence of pericentromeric heterochromatin (9qh-) in a patient with bilateral retinoblastoma. Acta Genet. Med. Gemellol. 46: 193-198.

Sumner AT (1982). The nature and mechanisms of chromosome banding. Cancer Genet. Cytogenet. 6: 59-87.

Tsuda H, Takarabe T, Kanai Y, Fukutomi T et al. (2002). Correlation of DNA hypomethylation at pericentric heterochromatin regions of chromomes 1 and 16 with histological features and chromosomal abnormalities of human breast carcinomas. Am. J. Pathol. 161: 859-866. 